\title{
Erratum to: Novel fibronectin-targeted nanodisk drug delivery system displayed superior efficacy against prostate cancer compared with nanospheres
}

Luyao Wang, Bingjie Zhou, Shiqi Huang, Mengke Qu, Qing Lin, Tao Gong, Yuan Huang, Xun Sun, Qin He, Zhirong Zhang, and Ling Zhang $(\triangle)$

Key Laboratory of Drug Targeting and Drug Delivery Systems, Ministry of Education, West China School of Pharmacy, College of Polymer Science and Engineering, Sichuan University, Chengdu 610041, China

(c) Tsinghua University Press and Springer-Verlag GmbH Germany, part of Springer Nature 2020

\section{Erratum to}

Nano Research 2019, 12(10): 2451-2459

https://doi.org/10.1007/s12274-019-2488-3

The description of nanodisk and nanosphere for their composing proportion in page 2 , Section 2.2 of the original version of this article was unfortunately reversed.

\section{Instead of}

The PEG-stabilized nanodisks (ND) consisted of POPC/ cholesterol/mPEG2000-DSPE (15:8:7, w/w/w). The CR(NMe)EKA peptide modified nanodisks (ND-P) consisted of POPC/ cholesterol/mPEG2000-DSPE/DSPE-PEG2000-Mal (15:8:3:4, $\mathrm{w} / \mathrm{w} / \mathrm{w} / \mathrm{w})$. The components of nanosphere without modification (NS) were POPC/cholesterol/mPEG2000-DSPE (7:4:19, w/w/w) and the components of modified nanosphere (NS-P) were POPC/cholesterol/mPEG2000-DSPE/DSPE-PEG2000-Mal $(7: 4: 15: 4, w / w / w / w)$.
It should read

The PEG-stabilized nanodisks (ND) consisted of POPC/ cholesterol/mPEG2000-DSPE (7:4:19, w/w/w). The CR(NMe)EKA peptide modified nanodisks (ND-P) consisted of POPC/ cholesterol/mPEG2000-DSPE/DSPE-PEG2000-Mal (7:4:15:4, $\mathrm{w} / \mathrm{w} / \mathrm{w} / \mathrm{w})$. The components of nanosphere without modification (NS) were POPC/cholesterol/mPEG2000-DSPE (15:8:7, w/w/w) and the components of modified nanosphere (NS-P) were POPC/cholesterol/mPEG2000-DSPE/DSPE-PEG2000-Mal $(15: 8: 3: 4, w / w / w / w)$. 